\title{
Universiteit
}

Leiden

The Netherlands

\section{Psychosocial stress impairs working memory at high loads: an association with cortisol levels and memory retrieval.}

Oei, N.Y.L.; Everaerd, W.; Elzinga, B.M.; Well, S. van; Bermond, B.

\section{Citation}

Oei, N. Y. L., Everaerd, W., Elzinga, B. M., Well, S. van, \& Bermond, B. (2006). Psychosocial stress impairs working memory at high loads: an association with cortisol levels and memory retrieval. Stress, 9, 133-141. Retrieved from https://hdl.handle.net/1887/14213

Version: $\quad$ Not Applicable (or Unknown)

License: $\quad$ Leiden University Non-exclusive license

Downloaded from: https://hdl.handle.net/1887/14213

Note: To cite this publication please use the final published version (if applicable). 


\title{
Psychosocial stress impairs working memory at high loads: An association with cortisol levels and memory retrieval
}

\author{
N. Y. L. OEI ${ }^{1}$, W. T. A. M. EVERAERD ${ }^{2}$, B. M. ELZINGA ${ }^{1}$, S. VAN WELL ${ }^{2}, \&$ B. BERMOND ${ }^{2}$ \\ ${ }^{1}$ Department of Psychology, Leiden University, Wassenaarseweg 52, 2300 RB, Leiden, The Netherlands, and ${ }^{2}$ Department \\ of Psychology, University of Amsterdam, Roetersstraat 15, 1018 WB, Amsterdam, The Netherlands
}

(Received 9 Fune 2006; revised 27 Fuly 2006; accepted 12 August 2006)

\begin{abstract}
Stress and cortisol are known to impair memory retrieval of well-consolidated declarative material. The effects of cortisol on memory retrieval may in particular be due to glucocorticoid (GC) receptors in the hippocampus and prefrontal cortex (PFC). Therefore, effects of stress and cortisol should be observable on both hippocampal-dependent declarative memory retrieval and PFC-dependent working memory (WM). In the present study, it was tested whether psychosocial stress would impair both WM and memory retrieval in 20 young healthy men. In addition, the association between cortisol levels and cognitive performance was assessed. It was found that stress impaired WM at high loads, but not at low loads in a Sternberg paradigm. High cortisol levels at the time of testing were associated with slow WM performance at high loads, and with impaired recall of moderately emotional, but not of highly emotional paragraphs. Furthermore, performance at high WM loads was associated with memory retrieval. These data extend previous results of pharmacological studies in finding WM impairments after acute stress at high workloads and cortisol-related retrieval impairments.
\end{abstract}

Keywords: Arousal, salivary cortisol, memory retrieval, working memory

\section{Introduction}

Acute high-levels of GCs (cortisol in humans) affect memory and cognition (Lupien and McEwen 1997; Wolf 2003). Cortisol or stress have been found to influence various forms of memory differently (e.g. Lupien et al. 1999; Vedhara et al. 2000), and in addition affect each memory phase differentially (Roozendaal 2000; 2002). Cortisol elevations immediately after learning have been shown to enhance declarative memory consolidation, specifically of material with emotionally arousing content (Buchanan and Lovallo 2001; Cahill et al. 2003; Kuhlmann and Wolf 2006). Conversely, the association between pre-retrieval stress or high cortisol levels and impaired memory retrieval has been reported consistently (de Quervain et al. 2000; 2003). Here too emotionally arousing and negatively valenced material appears to be more affected by high cortisol levels at the time of retrieval testing than neutral, non-arousing stimuli (Kuhlmann et al. 2005a,b; Buchanan et al. 2006).

The effects of cortisol on declarative memory retrieval have mainly been attributed to the actions of glucocorticoid (GC) receptors in the hippocampus (Roozendaal 2002) and the prefrontal cortex (PFC) (Lupien and Lepage 2001). Therefore, effects of cortisol on prefrontal-dependent memory, like working memory (WM), should be observable. Indeed, some studies found that acute elevations of exogenous glucocorticosteroids impaired WM, without affecting declarative memory (Lupien et al. 1999; Wolf et al. 2001). Lupien et al. (1999) infused hydrocortisone $(40,300$ or $600 \mu \mathrm{g} / \mathrm{dl} / \mathrm{kg})$ or placebo in young healthy men and assessed WM using an item-recognition task (Sternberg 1966) that consisted of trials with low to high comparison loads. Lupien et al. (1999) found that WM was affected at high comparison loads, indicated by slower reactions times for high- as compared with low-comparison loads. Cortisol was

Correspondence: N. Y. L. Oei, Section Clinical and Health Psychology, Department of Psychology, Wassenaarseweg 52, 2300 RB, Leiden, The Netherlands. Tel: 3171527 3836. Fax: 3171527 4678. E-mail: noei@fsw.leidenuniv.nl 
not associated with impaired declarative memory. However, both learning and retrieval took place after infusion of hydrocortisone, which made it difficult to draw conclusions with regard to cortisol effects on retrieval specifically, and in comparison with WM.

Although declarative memory encoding and consolidation are known to be dependent on the hippocampus, retrieval of declarative memory is also mediated by the PFC (Buckner and Wheeler 2001; Ranganath et al. 2003; Simons and Spiers 2003). Also, although WM tasks are known to depend on prefrontal brain areas, there is evidence from studies using magnetic encephalograms (MEG) (Campo et al. 2005) and functional magnetic resonance imaging (fMRI) (Ranganath and D'Esposito 2001; Karlsgodt et al. 2005) that the medial temporal lobe (MTL) is activated during WM tasks. Moreover, activity in the dorsolateral PFC has also been found during memory retrieval and $\mathrm{WM}$, possibly reflecting monitoring (Cabeza et al. 2002; Nyberg et al. 2003) or selection of task-relevant information (Sakai and Passingham, 2004). So far, one imaging $\left(\mathrm{H}_{2}^{15} \mathrm{O}-\right.$ positron emission tomography) study has shown cortisol-induced decreased blood flow in the MTL associated with impaired performance on a delayed recall task (de Quervain et al. 2003). A recent fMRIstudy, showed cortisol-induced decreased brain activation in both the PFC and hippocampus during declarative memory retrieval (Oei et al. submitted), which suggests that stress effects on retrieval may partly be caused by cortisol effects on prefrontal functioning.

Studies in which cortisol levels are elevated by psychosocial stress have seldom tested both WM and declarative memory retrieval. WM was tested in at least two psychosocial stress studies with the Wais- $R$ subtest Digit Span (DS): One reported impairing effects on memory retrieval associated with cortisol levels, but no impairing effects on WM (Kuhlmann et al. 2005b), the other did not assess memory retrieval and reported impairment on DS-forwards during stress (Elzinga and Roelofs 2005). However, DS-forwards is considered to be a measure of attention, whereas DS-backwards a test of WM (Ackerman et al. 2002). Furthermore, DS has been shown to be selectively preserved following frontal and hippocampal lesions in humans (Cave and Squire 1992; Daffner et al. 2000). Clearly, stress effects on WM in healthy individuals should be replicated with the use of more sensitive WM tasks.

The goal of the present study was to test whether high cortisol levels impair both WM and declarative memory retrieval in young healthy men and to assess the association between these two measures. In addition, it was examined whether cortisol differentially affects retrieval of material with different arousal properties.

\section{Method}

\section{Participants}

A total of 20 healthy male first-year psychology students participated in this study. All participants were informed about the study and gave written consent before participation and received obligatory course marks. Participants were screened before inclusion. Criteria for inclusion were: a body mass index $\left(\mathrm{BMI}=\mathrm{kg} / \mathrm{m}^{2}\right)$ between 19 and 25, a healthy medical and psychiatric history, determined by a brief version of the Amsterdam Biographical Interview (ABV; Wilde 1963) and the Dutch version of the Symptom Checklist-90 (SCL-90; (Arrindell and Ettema 1986). Exclusion criteria included use of medication or psychotropic drugs within 3 months prior to the test sessions, blood pressure over $140 / 90 \mathrm{mmHg}$, diabetes mellitus, current and past psychiatric problems, and the use of remedies containing corticosteroids. The study was approved by the Ethical Committee of the Department of Psychology of the University of Amsterdam. Characteristics of the sample were as follows (mean $\pm \mathrm{SD}$ ): Age, $21.86 \pm 3.89$ years; BMI, $21.44 \pm 1.57 \mathrm{~kg} / \mathrm{m}^{2}$; SCL-90, $115.24 \pm 20.88$, which falls in the "normal range" scoring "average" using normative ratings for a healthy population. No significant differences were found between groups with different order of stress for age $(F[1,19]=0.004 ; p=0.95$; BMI $F[1,19]=$ $3.02 ; p=0.1$; SCL-90 $(F[1,19]=1.07 ; p=0.31)$.

\section{Design}

Testing was done in a randomized crossover design on two consecutive ("retrieval") days at 09.30 AM, to ensure high basal endogenous cortisol levels. Although absolute cortisol rises in response to stress do not differ between AM and PM phase (Kudielka et al. 2004), the AM phase was chosen so that cortisol rises would more likely occupy GC receptors (Lupien and Lepage 2001; Maheu et al. 2005, and see Het et al. (2005), for a review on time of day effects). The Sternberg-based WM task described by Lupien et al. (1999) was used. All participants encoded paragraphs 1 day earlier and were randomly assigned to stress order (stress on retrieval day 1 or 2). Psychosocial stress was induced to elevate cortisol levels.

\section{Memory tasks}

Working memory. WM was measured using the same item-recognition task (Sternberg 1966) used and described extensively by Lupien et al. (1999). Similar tasks have been reported to significantly activate the dorsolateral PFC in neuroimaging studies (e.g. Veltman et al. 2003). The WM processing load is manipulated by varying the numbers of uppercase letters (1-4 targets) that have to be held in memory for later recognition, and 
by varying the number of letters (1-4 displayed) presented in the recognition display after a short delay (750-ms), which leads to a load of 2-16 comparisons. Participants had to press a "yes" button indicating they had recognized a target (present-target trials), or a "no" button, when no target letter was recognized (absenttarget trials). Only one target letter was present in the present-target trials. To ensure the task was not too easy, we randomly delivered blocks with differing loads instead of steadily increasing comparison loads. To avoid boredom, we decreased the number of trials from 300 to 240 (16 trials per each of 15 conditions). Stimulus software was developed at the Department of Psychology of the University of Amsterdam (http:// www.psy.uva.nl/Service/SG/Software/Home.html, selectSoftware and then WESP), which randomizes and presents stimuli, and records reaction times (RTs) and errors.

Declarative memory. The Wechsler Memory ScaleRevised Logical Memory test (Wechsler 1981) was used. This paragraph recall test was used as a valid and sensitive measure of declarative memory that has proved to be sensitive to cortisol effects in previous studies (Elzinga et al. 2005). According to the WMS-LM method, four paragraphs were constructed, containing 21 pieces of information, matched for difficulty. However, the emotionality of the content of two paragraphs was reduced (e.g. a story about a fire alarm was changed into a story about a fire drill) whereas two paragraphs were "emotionalized" (e.g. a student was beaten to death on his way to his final examinations, instead of only beaten). Recall percentage was computed as "(delayed recall/immediate recall) $\times$ 100 ". In an exit-interview, participants rated the subjective emotional content of the paragraphs on a 9points Likert scale ranging from 1 (not emotional at all) to 9 (extremely emotional). A Wilcoxon $t$-test for paired samples showed that participants rated the "moderately emotional" paragraphs (mean \pm SEM, $2.2 \pm 0.31$ ) as significantly less emotional than the "highly emotional" paragraphs (mean \pm SEM, $3.9 \pm 0.41)(z=-2.85$, $N$-ties $=16, p=0.002$, one-tailed). These means were similar to mean arousal ratings of "moderately emotional" and "highly emotional" words used by Buchanan et al. (2006).

\section{Psychosocial stress protocol}

To induce psychosocial stress, the Trier Social Stress Test (TSST) was employed (Kirschbaum et al. 1993). In male participants, the TSST protocol has consistently been shown to raise cortisol levels, in both saliva and blood. This laboratory stressor consists of a 10-min period in anticipation of a 5min free speech and a 5-min arithmetic task in front of a selection committee. The TSST protocol was followed, with the exception of the arithmetic task, which was exchanged by a "3-back only" task, to make the stressor even more difficult. A set of 100 randomly generated digits were presented aurally in a fixed order by the computer. Participants had to indicate whether each aurally-presented digit was similar to or different from the digit presented three digits back, by saying out loud "yes" to a target and "no" to a non-target. The task consisted of 30\% targets. One committee member responded to incorrect answers by saying out loud "incorrect", while another member kept up each participant's performance by means of a clearly visible scoreboard. When "incorrect", the scoreboard was ostentatiously put back to zero.

\section{Cardiovascular measures}

Systolic blood pressure (SBP, $\mathrm{mmHg}$ ), diastolic blood pressure $(\mathrm{DBP}, \mathrm{mmHg})$, and heart rate $(\mathrm{HR}, \mathrm{bpm})$ were recorded using a Finapres blood pressure monitor (Finapres 2300, Ohmeda, Englewood, CO, USA). The Finapres enables non-invasive continuous beat-to-beat monitoring of the finger arterial pressure waveform using a finger cuff applied to the middle phalanx of the middle finger (see also Imholz et al. 1998).

\section{Cortisol}

Cortisol was assayed in saliva samples collected with Salivettes (Sarstedt, Germany). Saliva sampling is a stress-free method to assess unbound cortisol (Kirschbaum and Hellhammer 1994). Saliva samples were centrifuged and thereafter stored at $-70^{\circ} \mathrm{C}$ until assayed. Free cortisol concentration in saliva was analyzed with a time-resolved immunoassay with fluorometric detection (as described in Dressendorfer et al. 1992). Inter- and intra-assay coefficients of variance were below 12 and $10 \%$, respectively. All saliva analyses were conducted at Prof Kirschbaum's Laboratory (http://biopsychologie.tu-dresden.de).

\section{Procedure}

Participants were invited to the laboratory on three consecutive days: an acquisition-day, a retrieval day with psychosocial stressor (stress), and a retrieval day without stress (control). Participants were randomly assigned to TSST on retrieval day 1 or TSST on retrieval day 2. On the acquisition-day, participants learned four paragraphs for immediate recall. Paragraph delivery was counterbalanced. On the first retrieval day, the appointment was scheduled at $09.00 \mathrm{~h}$. Participants had to refrain from food intake, sugar- or caffeine-containing drinks, and physical exercise at least $1.5 \mathrm{~h}$ before testing. Immediately after arrival, the first saliva sample was taken. The experimenter explained that all instructions and tasks would be provided on a computer screen and 
showed the appropriate response keys. She then went to an adjacent room and started the computerized protocol (using the VSRRP98 software package developed at the Department of Psychology, University of Amsterdam). Participants received all further instructions, questionnaires and tasks via the computer screen and provided all their responses by means of the response keys, except for the instruction and responses with regard to the 3-back only task. HR and blood pressure were recorded continuously using a Finapres 15 min before, during, and $10 \mathrm{~min}$ after the TSST. Participants were instructed to minimize all movement during the physiological recordings. After adaptation to the Finapres a 15-min baseline period followed in which participants watched a documentary about salt men in Tibet. After the TSSTa 10-min recovery period followed in which participants watched the second segment of the documentary. Hereafter the Finapres fingercuff was removed. Saliva samples were collected immediately after the baseline period, just before the anticipation of the stressor (t1), before the free speech (t2), immediately after the 3-back task ( $\mathrm{t} 3$ ), $10 \mathrm{~min}$ after the cessation of the TSST when peak levels are expected (t4) and WM testing starts $(09.55 \mathrm{~h})$, and finally, $30 \mathrm{~min}$ after the stress challenge at the end of declarative memory testing (t5). WM was tested immediately after the recovery period ( $t 4)$. Hereafter, delayed recall of two paragraphs (one with highly emotional and one with moderately emotional content) was administered. On the day without stress, participants filled in questionnaires until the WM task and the other two paragraphs (one highly emotional, the other moderately emotional) were administered at exactly the same time as after the stress procedure. Saliva was sampled at exactly the same time points $(t 4, t 5)$. Paragraph recall was balanced across retrieval days and across participants to avoid any non-random bias. Finally, participants completed an exit-interview, in which they were asked to assess the paragraphs and to indicate their impression and sentiments about the members of the selection committee.

\section{Statistics}

Data were analyzed using repeated measures ANOVAs. Data were checked for the sphericity assumption, and Greenhouse-Geisser corrections were applied when this assumption was not met. Follow-up analysis of ANOVA effects was done with $t$-tests. Pearson's Product Moment Correlations between cortisol level and memory performance were computed. The data were analysed using SPSS for Windows, version 11.5.

\section{Results}

Order effects

An ANOVA for RTs was performed with Order (stress on the first retrieval day vs. stress on the second retrieval day) as between-subjects factor, and Condition (stress vs. control), Target type (present vs. absent) and Comparison load ( 2 vs. 3 vs. 4 vs. 6 vs. 8 vs. 9 vs. 12 vs. 16) as within-subjects factors. The ANOVA for RTs showed a main effect of Order, $F(1,18)=5.11$, $p=0.036$, and a significant interaction-effect of Condition by Order, $F(1,18)=11.22, p=0.004$, indicating that learning under stress had a significantly negative effect on later (stress-free) performance, whereas stress-free learning appeared to facilitate later performance when stressed (see Figure 1). In contrast, a repeated measures ANOVA with Order (stress on retrieval day 1 vs. stress on retrieval day 2) as betweensubjects factor, and Condition (stress vs. control) and Arousal (low, high) as within-subjects factors performed on delayed recall of the paragraphs revealed no significant effect of Order, $F(1,18)=0.000$, $p=0.99)$, and no significant effect of Condition by Order, $F(1,18)=2.18, p=0.16$.

To be able to answer our original research questions, we chose to discard all data from the second retrieval day, because the WM data were free from carry-over effects only on the first retrieval day. Therefore, all further analyses are performed on the data from retrieval day 1 .

\section{Stress}

A repeated measures ANOVA with Time $(t 1-t 5)$ as within-subjects factor, showed that free cortisol concentrations in saliva increased significantly in response to exposure to the stress challenge (see Figure 2), with a significant effect of Time, $F(1.54 ; 13.83)=5.74$, $p=0.02$ (Greenhouse-Geisser corrected, $\varepsilon=0.38$ ). Then, a repeated measures ANOVA with Condition (stress vs. control) as between subjects factor, and time of cognitive testing $(\mathrm{t} 4, \mathrm{t} 5)$ as within subjects factor was performed. Here, a significant effect of Condition was found, $F(1,18)=6.59, p<0.02$, a significant within

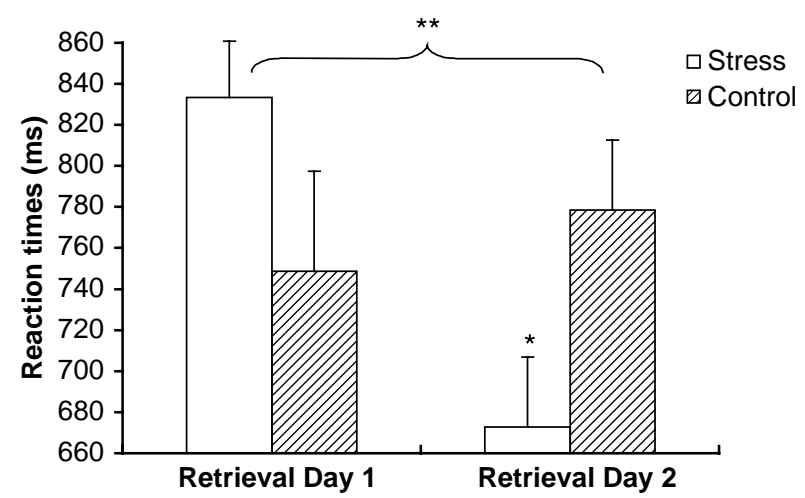

Figure 1. RTs mean (and SEM) of the groups in the WM task on two consecutive days. Stress on the first day significantly weakened the carry-over effect that was visible on the second day. The group that had stress on the first retrieval day was control group on the second retrieval day, and vice versa. * Faster WM performance in the stress group compared to the control group, $p<0.05$. $\star \star$ Significant interaction of Condition by Order, $p<0.005$. 


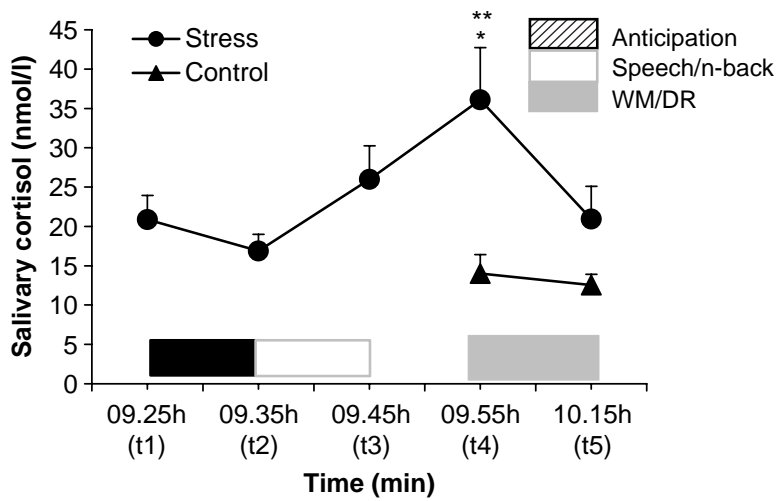

Figure 2. Salivary cortisol concentrations (means and SEM) in the stress group at five time points $(\mathrm{t} 1-\mathrm{t} 5)$ anticipating and responding to the TSST. Salivary cortisol levels of the control (no stress) group at two time points $(t 4, t 5)$, when cognitive testing was done, are shown for comparison. * Significant difference between cortisol levels at $\mathrm{t} 4$ and $\mathrm{t} 1$ in the stress group, $p<0.05$. $\star \star$ Significant difference between stress- and control group at $\mathrm{t} 4, p<0.05$.

subjects effect of time, and Time by condition ( $F \mathrm{~s}>10$, $p s<0.005)$, which were indicative of a decrease in cortisol level as time passed in the stress group. Independent $t$-tests showed that cortisol concentration just before the WM task (t4) was higher in the stress group (mean \pm SEM: $34.4 \pm 6.6 \mathrm{nmol} / 1)$, than in the control group (mean $\pm \mathrm{SEM}, 14 \pm 2.4 \mathrm{nmol} / \mathrm{l}$ ), $t_{11.37}=2.89, p=0.01$, whereas immediately after the declarative tests ( $\mathrm{t} 5$ ) the difference between cortisol level in the stress group (mean \pm SEM, $20.5 \pm 4.2 \mathrm{nmol} / \mathrm{l}$ ) and the control group (mean \pm SEM, 12.5 \pm $1.4 \mathrm{nmol} / \mathrm{l})$ was only a trend, $t_{10.97}=1.83, p=0.09$ (equal variances not assumed for both $t$-tests). Baseline cortisol levels of the groups (stress on day 1 , mean \pm $\mathrm{SEM}, 20.8 \pm 3.0 \mathrm{nmol} / \mathrm{l}$; stress on day 2 , mean $\pm \mathrm{SEM}$, $17.1 \pm 1.7 \mathrm{nmol} / \mathrm{l})$ did not differ significantly, $t_{14.21}=0.17$ (equal variances not assumed).

Separate repeated measures ANOVAs for SBP, DBP and HR, with time (anticipation through end of TSST) as within subjects variable showed significant elevations of these physiological measures during stress, for SBP, $F(3,27)=176.61, p<0.0005$; DBP, $F(1,4 ; 12,8)=$ $50.79, \quad p<0.0005 ; \quad H R, \quad F(1,2 ; 10,6)=17.38$, $p=0.001$ (see Table I). Planned comparisons between mean recovery and baseline of these measures were conducted using paired $t$-tests, which showed that HR had returned back to baseline, $t_{9}=-1.64, p>0.1$, in contrast to blood pressure, SBP, $t_{9}=-7.3, p<0.0005$; or DBP, $t_{9}=-3.6, p<0.01$. Additional post hoc $t$-tests showed that both of these measures had decreased significantly during the $10 \mathrm{~min}$ of recovery compared to mean stress levels during the TSST, SBP, $t_{9}=6.96$; DBP, $t_{9}=8.35(p s<0.0005)$.

\section{Memory performance}

Working memory. First, we inspected the data for errors. WM data of one participant from the stress group were excluded from this analysis, because of extreme numbers of detection errors and missing values due to no response within the maximum time $(>25 \%)$. A repeated measures ANOVA was performed with Condition (stress vs. control) as between subjects factor and Error type (present vs. absent) as within subjects factors. No main effect was found for Condition, $F(1,17)=0.25, p=0.63$, and confirm expectations, a significant main effect for Error type was found, reflecting more errors on present trials than on absent trials, $F(1,17)=73.83$; $p<0.0005$. No interaction-effect was found between Condition and Error type, $\mathrm{F}(1,17)=0.07, p>0.7$, or between Condition and Load, $F(7,119)=1.59$, $p>0.1$. There was, however, a near significant triple interaction of Condition by Load by Error type, $F(7$, $119)=2.06, p=0.05$, with more errors on present trials at high comparison loads in the stress group (mean $\pm \mathrm{SD}: 2.72 \pm 1.28$ ) compared to the control group (mean $\pm \mathrm{SD}$ : $1.93 \pm 1.03$ ).

Then, we performed a repeated measures ANOVA on RTs, to see if condition affected WM on different loads. There was no between-subjects effect of Condition, $F(1,17)=2.22, p=0.15$. A significant effect was found for Type, which reflected faster RTs for present trials than for absent trials, $F(1,17)=28.22, p<0.0005$. A main effect for

Table I. Blood pressure and HR before, during and after psychosocial stress $(n=10)$.

\begin{tabular}{|c|c|c|c|c|}
\hline & Time (h) & $\begin{array}{l}\mathrm{SBP}(\mathrm{mmHg}) \\
\text { Mean }(\mathrm{SD})\end{array}$ & $\begin{array}{c}\mathrm{DBP}(\mathrm{mmHg}) \\
\text { Mean }(\mathrm{SD})\end{array}$ & $\begin{array}{l}\text { HR (bpm) } \\
\text { Mean (SD) }\end{array}$ \\
\hline \multirow[t]{3}{*}{ Baseline } & 09.10 & $139.40(14.39)$ & $87.86(12.21)$ & $68.78(12.93)$ \\
\hline & 09.15 & $139.84(13.48)$ & $86.19(11.45)$ & $68.58(12.31)$ \\
\hline & 09.20 & $138.14(13.02)$ & $84.54(10.67)$ & $67.92(11.72)$ \\
\hline \multirow[t]{2}{*}{ Anticipation } & 09.25 & $150.03(17.39)$ & $89.84(13.16)$ & 72.87 (13.76) \\
\hline & 09.30 & $150.88(17.37)$ & $90.48(14.18)$ & $74.01(12.87)$ \\
\hline Speech & 09.35 & $195.23(18.94)$ & $117.66(19.96)$ & $89.96(14.78)$ \\
\hline 3-back & 09.40 & $189.31(24.77)$ & $113.07(22.59)$ & $82.02(17.43)$ \\
\hline \multirow[t]{2}{*}{ Recovery } & 09.45 & $167.14(24.99)$ & $101.23(21.38)$ & $69.91(10.80)$ \\
\hline & 09.50 & $163.89(25.90)$ & $99.13(20.36)$ & $69.09(10.84)$ \\
\hline
\end{tabular}

Values represent means and standard deviations (SD). SBP, systolic blood pressure (mmHg); DBP, diastolic blood pressure (mmHg); HR, heart rate (bpm, beats per minute). 
comparison load was found, $F(3.38 ; 57.52)=153.41$; $p<0.0005$, showing that higher comparison loads led to a linear increase of RTs. A significant Condition by Comparison load interaction was found, $F(3.38$; $57.52)=2.73, p=0.046$, with slower RTs in the stress group at higher comparison loads irrespective of type (see Figure 3). Additional one-tailed $t$-tests showed that the difference between stress and control group on high comparison loads was significant, for load $8\left(t_{14.72}=1.82, p=0.04\right)$, load $12\left(t_{14.34}=1.93\right.$, $p=0.04)$ and load $16\left(t_{10.22}=2.06, p=0.03\right)$ (other loads $p$ s $>0.1)$. Post hoc effect sizes were calculated using $r$ (Field, 2005, p. 294) which showed that these effects were large, $r=0.43, r=0.45$ and $r=0.54$, for load 8, 12 and 16, respectively, which indicates that the differences found between stress and control group were not likely due to a type I statistical error. Moreover, the increase in errors with higher loads in the stress group, was not a consequence of a speedaccuracy trade-off, since Pearson's correlations showed that longer RTs of Present trials at averaged high loads were positively associated with mean errors in the stress group $(r=0.65, p=0.06)$, but not in the control group $(r=0.09, p=0.82)$.

Cortisol and working memory. To see whether cortisol levels at the time of WM testing were associated with WM performance, Pearson's correlations were calculated between cortisol level ( $\mathrm{t} 4)$ and averaged RTs at low loads $(2,3,4,6)$ and high loads (load 8,12 , 16). No significant association was found at low loads $(r=0.08, p=0.37, n=19)$ or at high load ( $r=0.21$, $p=0.20, n=19$, both $p$ s one-tailed). When examining

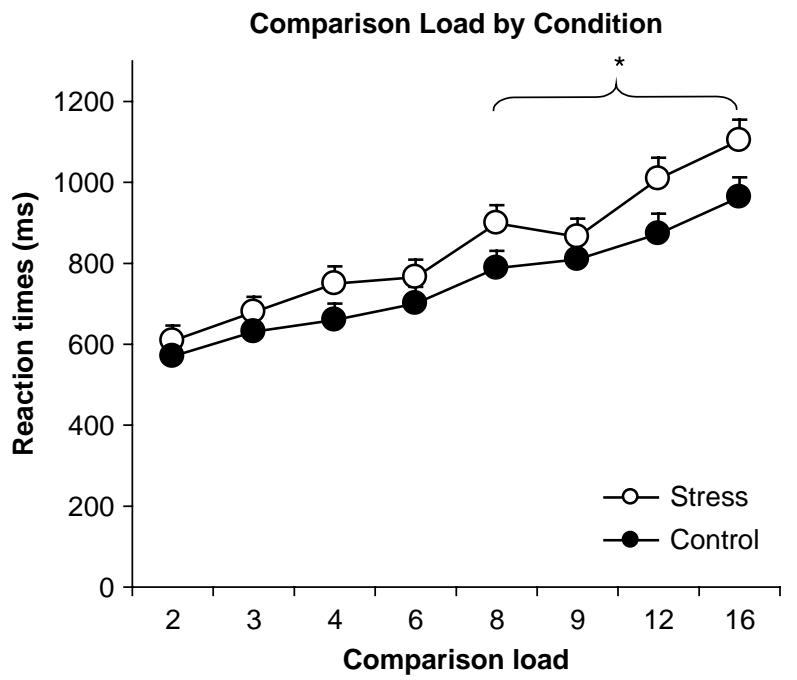

Figure 3. RTs (mean and SEM) of the two groups (stress and control, $n=19$ ) in the WM task as a function of comparison load. At high comparison loads the stress group was significantly slower than the control group. Notice that the RTs at comparison load 9 are faster, similar to the data of Lupien et al. (1999), which is probably because load 9 has fewer events, compared to other loads. ${ }^{\star} p<0.05$ (one-tailed). cortisol levels at the time of WM testing, two outliers were detected in the stress group with exceptionally high cortisol levels $(>60 \mathrm{nmol} / \mathrm{l})$. Without these outliers, no significant correlation was found at low loads $(r=0.33$, $p=0.10$, one-tailed), but at high loads, higher cortisol levels were significantly associated with slower RTs $(r=0.48, p=0.025 \text {, one-tailed })^{\dagger}$.

Declarative memory retrieval. The ANOVA performed on delayed recall of highly emotional (mean \pm SEM, stress: $48.26 \pm 9.14 \%$, and control: $56.65 \pm 7.62 \%$ ) and moderately emotional (mean \pm SEM, stress: $48.55 \pm 5.34 \%$; control, $50.20 \pm 6.74 \%$ ) paragraphs revealed no main effect of condition, or arousal (high, low), and no interaction of condition by arousal (all ps $>0.5)$.

Cortisol levels and memory retrieval. Pearson's correlations were calculated between cortisol level (t5) and paragraph recall. For the moderately emotional paragraph, a significant negative correlation was found $(r=-0.44, p=0.02$, one-tailed, $n=20)$. When inspecting the scatterplot, one outlier was observed, with extremely high cortisol level $(>50 \mathrm{nmol} / \mathrm{l})$. After removing the outlier the correlation was $r=-0.67$, $p=0.001$ (one-tailed, $n=19$ ), indicating that the higher the cortisol levels, the lower the score on moderately emotional paragraph recall (see Figure 4). No such association was found between cortisol level and recall of the highly emotional paragraphs $(r=0.17$, $p>0.23$, one-tailed $)^{\ddagger}$.

Working memory by memory retrieval. Pearson's correlations were calculated between WM performance at high comparison loads and moderately emotional

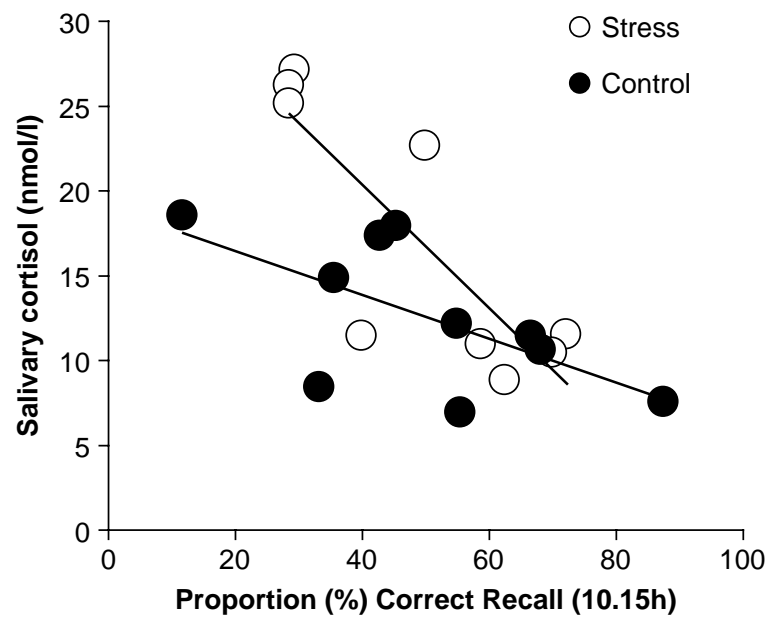

Figure 4. The association between the proportion correct recall of the moderately emotional paragraph and cortisol level at the time of testing. Higher cortisol levels were associated with less recall. In the stress group $(n=9)$, salivary cortisol concentration explained $69 \%$ of the variance in moderately emotional declarative memory recall (entire sample: $R^{2}=43.5 \%$ ). 
paragraph recall. A significant negative correlation was found between moderately emotional paragraph recall and high load $(8,12$ and 16$)(r=-0.57, p=0.01$, twotailed).

\section{Discussion}

The present study showed that psychosocial stress impaired WM performance at high but not low WM loads. High cortisol levels were associated with slower WM performance at high loads. In addition, a negative association between cortisol levels and delayed recall of moderately emotional material was found. Recall performance of the moderately emotional paragraphs was also associated with WM performance. No such association was found for highly emotional paragraphs. Together, the results of the present study extend the findings of pharmacological studies in finding WM impairments after acute stress, with moderate cortisol elevations.

The impairing effects of stress on WM performance at only high loads are consistent with the findings of Lupien et al (1999). Here, too, RTs were slower at high loads in the stress group only. However, the relative increase of cortisol levels in their study (mean $\pm 90 \mathrm{nmol} / \mathrm{l}$ ) differed to a great extent from levels found in our study (mean $\pm 12 \mathrm{nmol} / \mathrm{l}$ ). In our study, comparison loads were randomized to increase the difficulty of the WM task. This may have led to these highly similar results. In addition, in the present study, stress led to the tendency to erroneously indicate present targets at high loads as not previously encountered. These errors were associated with slower RTs. This bias toward rejection was specific for Present trials. There were no significantly less false hits in the Absent trials, so there was no indication of conservative responding in general. This tendency for more errors further corroborates the impairment in WM at high loads after acute stress.

An explanation that has been given in other studies for finding WM deficits (Elzinga and Roelofs 2005) or declarative memory retrieval deficits (Kuhlmann et al. $2005 \mathrm{~b}$ ) is stronger adrenergic activation due to the psychosocial stress. Rat studies have shown that corticosterone interacts with adrenergic mechanisms in the amygdala and hippocampus in causing retrieval impairments (Roozendaal et al. 2004). In humans, Elzinga and Roelofs (2005) did not find WM impairments (DS-forwards), $30 \mathrm{~min}$ after the TSST had finished and sympathetic activation had subsided, but only during the psychosocial stress (although the stress context was removed only $15 \mathrm{~min}$ before WM testing). We started WM testing $10 \mathrm{~min}$ after the psychosocial stressor. Unfortunately we were not able to proceed with the continuous cardiovascular measurements during the WM task, due to the fact that RTs tasks require speedy hand movements, which interfere with blood pressure assessments, and produce movement artefacts. Salivary cortisol concentration was peaking at the start of the WM task, and HR had returned to baseline. However, although blood pressure was significantly lower at that time than during stress, it was still significantly elevated indicating some sympathetic activation was present during WM testing. Moreover, it can be argued that the task itself could have induced acute increases in sympathetic activation, particularly at high comparison loads that are very demanding and perhaps frustrating. If this was the case, then sympathetic activation would also have been increased during performance at low loads, since trials at high and low comparison loads were delivered randomly. This would imply that high sympathetic activation and high cortisol levels do not impair WM performance at low comparison loads, in contrast to high load performance. Taken together, the present data cannot definitely answer the question whether stress-induced WM impairments require concurrent (very) high sympathetic activation. Clearly, more WM studies are warranted to investigate the differential effects of sympathetic activations and cortisol at different workloads.

In line with a recent study (Buchanan et al. 2006), high salivary cortisol levels in the present study were associated with less recall of moderately emotional, but not of highly emotional paragraphs. Buchanan et al. (2006) found retrieval impairments associated with cortisol elevations in responders to the cold pressor test. Moderately arousing words learned $1 \mathrm{~h}$ before elevation of cortisol levels, were recalled less well than highly arousing or neutral words. One possible explanation for this finding is that the memory trace of emotionally highly arousing material is more stable and thus less vulnerable to the modulatory effects of cortisol than moderately arousing material. However, our results should be interpreted with caution. First, mean recall of highly emotional paragraphs was clearly reduced after stress, but individual differences in recall of the highly emotional paragraphs were large in both stress and control group. Second, it was not assessed to what extent encoding was affected by the arousal properties of the material. Third, we could not compare these findings with recall of neutral, nonarousing stimuli. Human data on the interaction of stress or GCs and arousing stimuli (or material with different valence) are sparse and far from consistent. For instance, Domes et al. (2004) found that stress impaired the retrieval of positive words, but not of neutral or negative words. Kuhlmann et al. (2005a,b) found (a trend towards) retrieval impairment for positive and negative words after cortisol or stress treatment. Buss et al. (2004) found significant impairment in retrieval of neutral autobiographical episodes in young men, and only a trend for impaired retrieval of positive or negative episodes. Differences in timing, tasks and gender of the participants may be 
the reason for the divergence in direction of cortisol effects on memory retrieval of material with different valence and arousal properties (Wolf et al. 2004; Maheu et al. 2005).

According to our expectations, impaired WM performance at high loads was associated with low retrieval performance. Since we did not assess intelligence, it is possible that the association between WM and retrieval impairment reflects an underlying variance in intelligence levels between the groups. Nonetheless, the sample came from a university population and the allocation to groups was random, which may have reduced the chance of large differences in IQ variance. Moreover, performance on both measures was also associated with cortisol level. Cortisol may have parallel effects on the structures on which WM and memory retrieval are known to rely, the PFC and MTL, and this way independently affect WM and memory retrieval. However, there is evidence from imaging studies that show common activity of the MTL and the PFC during retrieval and WM (Buckner and Wheeler, 2001; Ranganath and D'Esposito, 2001; Cabeza et al. 2002; Nyberg et al. 2003; Ranganath et al. 2003; Simons and Spiers, 2003; Sakai and Passingham, 2004; Karlsgodt et al. 2005), and cortisol-induced decreases in those areas (Oei et al. submitted). Therefore, it could be speculated that apart from direct effects on specific areas cortisol impairs memory indirectly through general effects on a frontotemporal network. Low loads from the Sternberg paradigm have been associated with activations in the left ventrolateral PFC, but at high loads with right dorsolateral PFC activation (Manoach et al. 1997; Bunge et al. 2001). The latter area is linked to episodic memory retrieval (Cabeza et al. 2002). This suggests that of the two subprocesses of WM, "manipulation" might be more sensitive to the effects of cortisol and stress, as opposed to "maintenance" processes. These domains await further research using imaging techniques.

Many brain activations attributed to specific cognitive processes probably reflect general processes (Cabeza et al. 2003). Cabeza et al. (2003) found a common network for episodic memory retrieval and attention. They suggest that "post-retrieval monitoring" as an interpretation for these PFC activations should be rephrased in terms of attentional processes. Selective attention was not assessed in our study. However, there is evidence that cortisol impedes selective attention, leading to lower sensory acuity (Fehm-Wolfsdorf et al. 1993), and stress-induced high cortisol levels have been associated with decreased inhibition of non-relevant information on a negative priming task, a standard measure of inhibitory attentional processes (Skosnik et al. 2000). However, it still remains to be determined whether stress impairs memory retrieval through its effects on general attentional processes.
Taken together, these findings further substantiate the effects of stress and cortisol on memory functioning. Specifically, we found that stress impairs WM at high loads, but not low loads. Our sample was small and therefore conclusions should be made with caution. However, our results on the WM task converge with the findings of Lupien et al. (1999), which increases the validity of our findings. Future studies should use sensitive measures of WM and attention, when investigating effects of stress or cortisol on memory retrieval. In addition, stimuli with different arousal properties and their valence should be carefully employed when investigating the effects of stress or cortisol on memory.

\section{Notes}

${ }^{\dagger}$ To allow for a better comparison with previous work by others, cognitive performance was associated with cortisol levels calculated with the area under the curve method (AUCg: see Pruessner et al. 2003, for details on this measure) and with delta increase, which could only be provided for the stress group $(n=9)$. For cortisol level (in log AUCg) and low loads, $r=-0.62, p=0.04$, with high loads, $r=-0.59, p=0.04$. For delta increase and low loads, $r=-0.14, p=0.36$; with high loads, $r=-0.13, r=0.37$ (all onetailed).

${ }^{\ddagger}$ Cortisol level (log cortisol AUCg) in the stress group $(n=10)$ correlated significantly with retrieval of the moderately emotional paragraph, $r=-0.64, p=0.02$ (one-tailed), but not with the highly emotional paragraph, $r=0.05, p=0.44$ (one-tailed). Paragraph recall did not correlate significantly with cortisol when delta increase ( $\mathrm{t} 5-\mathrm{t} 1)$ was used as a measure (both $p \mathrm{~s}>0.3$, onetailed).

\section{References}

Ackerman PL, Beier ME, Boyle MO. 2002. Individual differences in working memory within a nomological network of cognitive and perceptual speed abilities. J Exp Psychol Gen 131:567-589.

Arrindell WA, Ettema JHM. 1986. SCL-90. Handleiding bij een multidimensionele psychopathologie-indicator. Lisse: Swets \& Zeitlinger B.V.

Buchanan TW, Lovallo WR. 2001. Enhanced memory for emotional material following stress-level cortisol treatment in humans. Psychoneuroendocrinology 26:307-317.

Buchanan TW, Tranel D, Adolphs R. 2006. Impaired memory retrieval correlates with individual differences in cortisol response but not autonomic response. Learn Mem 13:382-387.

Buckner RL, Wheeler ME. 2001. The cognitive neuroscience of remembering. Nat Rev Neurosci 2:624-634.

Bunge SA, Ochsner KN, Desmond JE, Glover GH, Gabrieli JD. 2001. Prefrontal regions involved in keeping information in and out of mind. Brain 124:2074-2086.

Buss C, Wolf OT, Witt J, Hellhammer DH. 2004. Autobiographic memory impairment following acute cortisol administration. Psychoneuroendocrinology 29:1093-1096.

Cabeza R, Dolcos F, Graham R, Nyberg L. 2002. Similarities and differences in the neural correlates of episodic memory retrieval and working memory. Neuroimage 16:317-330.

Cabeza R, Dolcos F, Prince SE, Rice HJ, Weissman DH, Nyberg L. 2003. Attention-related activity during episodic memory retrieval: A cross-function fMRI study. Neuropsychologia 41:390-399.

Cahill L, Gorski L, Le K. 2003. Enhanced human memory consolidation with post-learning stress: Interaction with the degree of arousal at encoding. Learn Mem 10:270-274.

Campo P, Maestu F, Capilla A, Fernandez S, Fernandez A, Ortiz T. 2005. Activity in human medial temporal lobe associated with 
encoding process in spatial working memory revealed by magnetoencephalography. Eur J Neurosci 21:1741-1748.

Cave CB, Squire LR. 1992. Intact verbal and nonverbal short-term memory following damage to the human hippocampus. Hippocampus 2:151-163.

Daffner KR, Mesulam MM, Holcomb PJ, Calvo V, Acar D, Chabrerie A, Kikinis R, Jolesz FA, Rentz DM, Scinto LF. 2000. Disruption of attention to novel events after frontal lobe injury in humans. J Neurol Neurosurg Psychiatry 68:18-24.

de Quervain DJ, Roozendaal B, Nitsch RM, McGaugh JL, Hock C. 2000. Acute cortisone administration impairs retrieval of longterm declarative memory in humans. Nat Neurosci 3:313-314.

de Quervain DJ, Henke K, Aerni A, Treyer V, McGaugh JL, Berthold T, Nitsch RM, Buck A, Roozendaal B, Hock C. 2003. Glucocorticoid-induced impairment of declarative memory retrieval is associated with reduced blood flow in the medial temporal lobe. Eur J Neurosci 17:1296-1302.

Domes G, Heinrichs M, Rimmele U, Reichwald U, Hautzinger M. 2004. Acute stress impairs recognition for positive words-association with stress-induced cortisol secretion. Stress 7:173-181.

Dressendorfer RA, Kirschbaum C, Rohde W, Stahl F, Strasburger CJ. 1992. Synthesis of a cortisol-biotin conjugate and evaluation as a tracer in an immunoassay for salivary cortisol measurement. J Steroid Biochem Mol Biol 43:683-692.

Elzinga BM, Roelofs K. 2005. Cortisol-induced impairments of working memory require acute sympathetic activation. Behav Neurosci 119:98-103.

Elzinga BM, Bakker A, Bremner JD. 2005. Stress-induced cortisol elevations are associated with impaired delayed, but not immediate recall. Psychiatry Res 134:211-223.

Fehm-Wolfsdorf G, Soherr U, Arndt R, Kern W, Fehm HL, Nagel D. 1993. Auditory reflex thresholds elevated by stress-induced cortisol secretion. Psychoneuroendocrinology 18:579-589.

Field A. 2005. Discovering statistics using SPSS. Thousand Oakes, New Delhi London: Sage Publications.

Het S, Ramlow G, Wolf OT. 2005. A meta-analytic review of the effects of acute cortisol administration on human memory. Psychoneuroendocrinology 30:771-784.

Imholz BP, Wieling W, van Montfrans GA, Wesseling KH. 1998. Fifteen years experience with finger arterial pressure monitoring: Assessment of the technology. Cardiovasc Res 38:605-616.

Karlsgodt KH, Shirinyan D, Van Erp TG, Cohen MS, Cannon TD. 2005. Hippocampal activations during encoding and retrieval in a verbal working memory paradigm. Neuroimage 25:1224-1231.

Kirschbaum C, Pirke KM, Hellhammer DH. 1993. The 'Trier Social Stress Test' $-\mathrm{a}$ tool for investigating psychobiological stress responses in a laboratory setting. Neuropsychobiology 28:76-81.

Kirschbaum C, Hellhammer DH. 1994. Salivary cortisol in psychoneuroendocrine research: Recent developments and applications. Psychoneuroendocrinology 19:313-333.

Kudielka BM, Schommer NC, Hellhammer DH, Kirschbaum C. 2004. Acute HPA axis responses, heart rate, and mood changes to psychosocial stress (TSST) in humans at different times of day. Psychoneuroendocrinology 29:983-992.

Kuhlmann S, Kirschbaum C, Wolf OT. 2005a. Effects of oral cortisol treatment in healthy young women on memory retrieval of negative and neutral words. Neurobiol Learn Mem 83:158-162.

Kuhlmann S, Piel M, Wolf OT. 2005b. Impaired memory retrieval after psychosocial stress in healthy young men. J Neurosci 25 : 2977-2982.

Kuhlmann S, Wolf OT. 2006. Arousal and cortisol interact in modulating memory consolidation in healthy young men. Behav Neurosci 120:217-223.

Lupien SJ, McEwen BS. 1997. The acute effects of corticosteroids on cognition: Integration of animal and human model studies. Brain Res Brain Res Rev 24:1-27.

Lupien SJ, Gillin CJ, Hauger RL. 1999. Working memory is more sensitive than declarative memory to the acute effects of corticosteroids: A dose-response study in humans. Behav Neurosci 113:420-430.

Lupien SJ, Lepage M. 2001. Stress, memory, and the hippocampus: Can't live with it, can't live without it. Behav Brain Res 127: 137-158.

Maheu FS, Collicutt P, Kornik R, Moszkowski R, Lupien SJ. 2005. The perfect time to be stressed: A differential modulation of human memory by stress applied in the morning or in the afternoon. Prog Neuropsychopharmacol Biol Psychiatry 29:1281-1288.

Manoach DS, Schlaug G, Siewert B, Darby DG, Bly BM, Benfield A, Edelman RR, Warach S. 1997. Prefrontal cortex fMRI signal changes are correlated with working memory load. Neuroreport 8:545-549.

Nyberg L, Marklund P, Persson J, Cabeza R, Forkstam C, Petersson KM, Ingvar M. 2003. Common prefrontal activations during working memory, episodic memory and semantic memory. Neuropsychologia 41:371-377.

Oei NYL, Elzinga BM, Wolf OT, de Ruiter MB, Damoiseaux JS, Kuijer JPA, Veltman DJ, Scheltens PH, Rombouts SARB. Cortisol decreases hippocampal and prefrontal activation during declaritive memory retrieval in young men. Submitted for publication.

Pruessner JC, Kirschbaum C, Meinlschmid G, Hellhammer DH. 2003. Two formulas for computation of the area under the curve represent measures of total hormone concentration versus timedependent change. Psychoneuroendocrinology 28:916-931.

Ranganath C, D'Esposito M. 2001. Medial temporal lobe activity associated with active maintenance of novel information. Neuron 31:865-873.

Ranganath C, Johnson MK, D’Esposito M. 2003. Prefrontal activity associated with working memory and episodic long-term memory. Neuropsychologia 41:378-389.

Roozendaal B. 2000. Curt P. Richter award. Glucocorticoids and the regulation of memory consolidation. Psychoneuroendocrinology 25:213-238.

Roozendaal B. 2002. Stress and memory: Opposing effects of glucocorticoids on memory consolidation and memory retrieval. Neurobiol Learn Mem 78:578-595.

Roozendaal B, Hahn EL, Nathan SV, de Quervain DJ, McGaugh JL. 2004. Glucocorticoid effects on memory retrieval require concurrent noradrenergic activity in the hippocampus and basolateral amygdala. J Neurosci 24:8161-8169.

Sakai K, Passingham RE. 2004. Prefrontal selection and medial temporal lobe reactivation in retrieval of short-term verbal information. Cereb Cortex 14:914-921.

Simons JS, Spiers HJ. 2003. Prefrontal and medial temporal lobe interactions in long-term memory. Nat Rev Neurosci 4:637-648.

Skosnik PD, Chatterton RT, Jr, Swisher T, Park S. 2000. Modulation of attentional inhibition by norepinephrine and cortisol after psychological stress. Int J Psychophysiol 36:59-68.

Sternberg S. 1966. High-speed scanning in human memory. Science 153:652-654.

Vedhara K, Hyde J, Gilchrist ID, Tytherleigh M, Plummer S. 2000. Acute stress, memory, attention and cortisol. Psychoneuroendocrinology 25:535-549.

Veltman DJ, Rombouts SARB, Dolan RJ. 2003. Maintenance versus manipulation in verbal working memory revisited: An fMRI study. Neuroimage 18:247-256.

Wechsler D. 1981. The Wechsler adult intelligence scale-revised. New York: Harcourt Brace Jovanovich, Inc.

Wilde GJS. 1963. Neurotische labiliteit gemeten volgens de vragenlijst methode. Amsterdam: Van Rossen 1963.

Wolf OT, Convit A, McHugh PF, Kandil E, Thorn EL, De Santi S, McEwen BS, de Leon MJ. 2001. Cortisol differentially affects memory in young and elderly men. Behav Neurosci 115:1002-1011.

Wolf OT. 2003. HPA axis and memory. Best Pract Res Clin Endocrinol Metab 17:287-299.

Wolf OT, Kuhlmann S, Buss C, Hellhammer DH, Kirschbaum C. 2004. Cortisol and memory retrieval in humans: Influence of emotional valence. Ann NY Acad Sci 1032:195-197. 